\title{
Effects of Insulin on Lipid Metabolism of Larvae and Metamorphosing Landlocked Sea Lamprey, Petromyzon marinus
}

\author{
Yung-hsi Kao, ${ }^{*, 1}$ John H. Youson, $\uparrow$ John A. Holmes, $\uparrow$ Azza Al-Mahrouki, $\uparrow$ \\ and Mark A. Sheridan* \\ * Department of Zoology and Regulatory Bioscience C enter, N orth Dakota State U niversity, Fargo, N orth Dakota \\ 58105-5517; and †D epartment of Zoology and Division of Life Sciences, U niversity of Toronto at Scarborough, \\ Scarborough, Ontario M 1C 1A4, Canada
}

Accepted January 25, 1999

This study was designed to examine the role of insulin (INS) in regulating changes in lipid metabolism of larval and metamorphosing landlocked lamprey, Petromyzon marinus. Larvae and stage 6 metamorphosing individuals were injected intraperitoneally once per day for 2 days with either saline (0.6\%), bovine INS (100 ng/g body weight), or alloxan ( $0.2 \mathrm{mg} / \mathrm{g}$ body weight). Insulin administration resulted in depressed plasma fatty acid (FA) levels, whereas alloxan injection elevated plasma FA levels at both life cycle intervals. In larvae, INSinduced hypolipidemia was attended by increased lipid concentration in kidney and muscle, reduced rates of lipolysis in kidney, liver, and muscle (as indicated by decreased triacylglycerol lipase activity), and, to a lesser extent, by higher rates of lipogenesis in kidney and muscle (as evidenced by higher acetyl-C oA carboxylase and/or diacylglycerol acyltransferase activities). In general, the effects of alloxan were opposite of those of INS. The alloxan-induced increase in plasma FA was supported by an enhanced rate of lipolysis in the kidney, a relatively lower rate of fatty acid synthesis in kidney, liver, and muscle, and a relatively lower renal rate of TG synthesis. In stage 6 metamorphosing lamprey, the INS-induced decline in plasma FA was attended by

\footnotetext{
${ }^{1}$ Current address: Ben May Institute for Cancer Research, University of Chicago, Chicago, IL 60637.
}

reduced renal and hepatic rates of lipolysis and by enhanced lipogenesis, as indicated by increased renal and hepatic rates of de novo fatty acid synthesis and hepatic and muscular rates of TG synthesis. In contrast, the increase in plasma FA induced by alloxan in stage 6 animals was supported by reduced TG synthsis in liver. Immunocytochemistry revealed that alloxan was not cytotoxic to pancreatic $\beta$ cells, suggesting that the effects of alloxan were extrapancreatic in the time frame of our study. Because insulin-induced lipogenesis and antilipolysis is similar to the pattern of lipid metabolism (phase I) displayed by lamprey during their spontaneous metamorphosis, INS may play a role, possibly in concert with other factors, in coordinating metamorphosis-associated changes in lipid metabolism. @ 1999 Academic Press

Key Words: insulin; lipolysis; lipogenesis; lamprey; Petromyzon marinus; metamorphosis.

The development of lamprey includes a 7-stage metamorphic episode which transforms microphagic filter-feeding larvae into either nonparasitic or parasitic adults, depending on species (e.g., nonparasitic Lampetra planeri and parasitic Petromyzon marinus) (Hardisty, 1979). During metamorphosis, the transforming larvae do not feed and undergo a vast array of morphological, physiological, and biochemical changes (Youson, 1980). Changes in lipid metabo- 
lism are among the most striking biochemical changes which accompany lamprey metamorphosis (for review see Sheridan and Kao, 1998); however, the systems responsible for regulating such changes are not known.

A role for insulin (INS) in the regulation of lipid metabolism in lamprey was first reported by Plisetskaya and Mazina (1969), who showed that injection of adult Baltic lamprey, Lampetra fluviatilis, with mammalian INS resulted in reduced plasma fatty acid (FA) levels. A lipogenic role of INS was further supported by the finding that injection of antiserum to mammalian INS elevated plasma FA levels in adult lamprey (Plisetskaya, 1980). Whether or not INS plays a role in regulating lipid metabolism during larval life or during lamprey metamorphosis is not known. The observation that plasma INS levels change significantly during various phases of lamprey life cycle, including higher levels in larvae than stage 4 metamorphosing animals (Youson et al., 1994), suggests that INS may regulate aspects of lipid metabolism in larval and metamorphosing individuals as well as in adults.

In this study, we used the landlocked form of sea lamprey, P. marinus, to examine the role of INS in regulating lipid metabolism in larvae and metamorphosing lamprey. We evaluated the effects of INS on plasma fatty acids as well as on activities of key lipolytic and lipogenic enzymes in kidney, liver, muscle, and intestine by exogenous administration of INS as well as by treatment with alloxan, a putative pancreatic $\beta$ cell cytotoxin.

\section{MATERIALS AND METHODS}

\section{Experimental Animals}

Sea lamprey, P. marinus, larvae were first collected from Oshawa Creek, a tributary of Lake Ontario, Ontario, Canada on May 31 and June 1, 1996, and transported to the University of Toronto at Scarborough, Ontario, Canada where they were fed baker's yeast once a week (18 g/25 larvae). Animals were maintained in 21 L glass aquaria with a sandy substrate and $12 \mathrm{~L}$ of dechlorinated City of Toronto water at an ambient temperature of $15-18^{\circ}$ under a photoperiod of $15 \mathrm{~h}$ light and $9 \mathrm{~h}$ dark.

\section{In Vivo Injection}

Larvae and stage 6 metamorphosing animals were segregated and placed into separate aquaria (10 larvae/ tank; 5 stage- 6 metamorphosing animals/tank; initial body length $124.7 \pm 1.3 \mathrm{~mm}$, body weight $2.74 \pm$ 0.07 , condition factor $1.412 \pm 0.019) 1$ week before experimentation. Feeding was suspended 2 days prior to experimentation. Lampreys were anesthetized by immersion in buffered $0.05 \%(\mathrm{w} / \mathrm{v})$ tricaine methanesulfonate (MS-222) and injected intraperitoneally $(10 \mu \mathrm{l} / \mathrm{g}$ body weight) with either $0.6 \%$ of saline (control), bovine INS (25.7 I.U./mg INS, Sigma I 5500 ) at a dose of $100 \mathrm{ng}$ INS (dissolved in $0.6 \%$ saline at $\mathrm{pH} 4.0) / \mathrm{g}$ body weight, or alloxan (5,6-dioxyuracil monohydrate; Sigma) at a dose $0.2 \mathrm{mg}$ alloxan (dissolved in $0.6 \%$ saline)/g body weight once per day for a 2-day period. This treatment regime was based on preliminary studies that showed that heterologous hormone (necessitated by the nature of our in vivo study) was effective at lowering both plasma glucose and plasma FA and that alloxan treatment elevated the concentrations of these metabolites. During the period of experimentation from 18 September 1996 to 20 September 1996, water and sand substrate in the aquaria were not changed. Twelve to $14 \mathrm{~h}$ after the last injection, animals were anesthetized individually with buffered MS-222 as described above and were weighed and measured. Blood was collected into heparinized-capillary tubes from the severed caudal vasculature, allowed to clot overnight at $4^{\circ}$, and then centrifuged (6000g for $5 \mathrm{~min}$ ). Plasma was collected and stored at $-70^{\circ}$ for later determination of fatty acids. Tissues (liver, kidney, muscle, and intestine) were removed, frozen in liquid $\mathrm{N}_{2}$, and then stored at $-70^{\circ}$ for later determination of total lipid and enzyme activities.

In a separate experiment, the alloxon-injection protocol described above was repeated at a later date on five larvae. Controls consisted of five uninjected and five saline-injected larvae. All animals were anesthetized with MS-222 as before, killed by decapitation, and their intestine, liver, and accompanying islet tissue were removed and placed in Bouin's fluid for $24 \mathrm{~h}$. The 
samples were immediately processed for light microscopy as described below.

\section{Biochemical Analysis}

Plasma fatty acids were measured by the micromethod of Noma et al. (1973). Total lipids and enzyme activities in the selected tissues, liver, kidney, muscle, and intestine, were extracted and analyzed as described by Kao et al. (1997a). The protein content of the enzyme preparations was determined in duplicate by the dye-binding method (Bradford, 1976) using a Bio-Rad (Richmond, CA) microplate reader and bovine serum albumin (Sigma, St. Louis, MO) as a standard. The rate of lipid breakdown was evaluated by assessing triacylglycerol lipase (TGL; EC 3.1.1.2) activity. Units of enzyme activity are expressed as picomoles of $\left[{ }^{3} \mathrm{H}\right]$ oleic acid released $/ \mathrm{h} / \mathrm{mg}$ protein from the $\left[{ }^{3} \mathrm{H}\right]$ triolein (tri- $\left[9,10(n)-{ }^{3} \mathrm{H}\right]$ oleate glycerol). The rate of lipid synthesis was evaluated by assessing separately de novo fatty acid synthesis, as determined by acetyl-CoA carboxylase (ACC; EC 6.4.1.2) activity, and triacylglycerol synthesis, as determined by diacylglycerol acyltransferase (DGAT; EC 2.3.1.20) activity. Units of ACC activity and DGAT activity are expressed as femtomoles of $\left[{ }^{14} \mathrm{C}\right]$ malonyl-CoA formed $/ \mathrm{h} / \mathrm{mg}$ protein and picomoles of $\left[{ }^{14} \mathrm{C}\right] \mathrm{TG}$ formed $/ \mathrm{min} / \mathrm{mg}$ protein, respectively.

\section{Immunocytochemistry}

The tissue samples containing islet tissue were embedded in paraffin and seven 5- $\mu$ m longitudinal sections were immunostained for INS using antibovine INS antiserum prepared in guinea pig (courtesy of Prof. C. Yip, University of Toronto). The Histostain Plus staining kit (Zymed Laboratories, Inc., San Francisco, CA), a labeled (strept) avidin-biotin method, was used. The antiserum was applied at a dilution of 1:1000 and negative controls consisted of replacing the primary antiserum with phosphatebuffered saline.

\section{Statistical Analysis}

Data are expressed as means \pm SEM. Analysis of variance (ANOVA) and Duncan's multiple range test were used to examine differences among the various groups. A probability level of 0.05 was used to indicate significance. All statistics were performed using SigmaStat (Jandel Scientific, Palo Alto, CA).

\section{RESULTS}

\section{Body Characteristics}

Body characteristics, including body weight, body length, condition factor, and tissue (liver, kidney, muscle, and intestine) weight, of larval and metamorphic lampreys were not affected by INS or alloxan treatment over the course of the 2-day experiment (Table 1).

\section{Plasma Fatty Acid Concentration}

The plasma levels of fatty acids (FA) in larvae and stage 6 transformers were significantly altered by INS and alloxan administration (Fig. 1). Insulin significantly reduced plasma FA levels by $48 \%$ in larvae and by $42 \%$ in stage 6 metamorphosing animals. Alloxan significantly elevated plasma FA levels by $72 \%$ in larvae and by $55 \%$ in stage 6 metamorphosing animals.

\section{Tissue Lipid Concentration}

In larvae, INS significantly increased lipid concentrations in kidney and muscle (Fig. 2). Following alloxan injection, lipid concentrations were generally similar to those in tissues removed from control animals. In stage 6 metamorphosing lamprey, lipid concentrations in the kidney, liver, muscle, and intestine were all similar to control values after either INS or alloxan administrations (Fig. 2).

\section{The Rate of Lipolysis}

The rate of lipolysis was assessed by changes in triacylglycerol (TG) lipase activity (Fig. 3). In larvae, INS generally decreased the rate of lipolysis; significantly reduced lipase activity was observed in liver and muscle. Treatment of larvae with alloxan increased, but not significantly so, renal lipolysis when 
TABLE 1

In Vivo Effects of Mammalian Insulin and Alloxan on Body Characteristics of Larvae and Stage 6 Metamorphosing Lampreys, P. marinus ${ }^{a}$

\begin{tabular}{|c|c|c|c|c|c|c|}
\hline \multirow[b]{2}{*}{ Parameters } & \multicolumn{3}{|c|}{ Larval lamprey } & \multicolumn{3}{|c|}{ Stage 6 metamorphosing lamprey } \\
\hline & Control & Insulin & Alloxan & Control & Insulin & Alloxan \\
\hline Body weight (g) & $2.85 \pm 0.10$ & $2.91 \pm 0.13$ & $2.87 \pm 0.10$ & $3.60 \pm 0.32$ & $3.37 \pm 0.19$ & $3.98 \pm 0.45$ \\
\hline Body length (cm) & $124.6 \pm 1.4$ & $125.4 \pm 2.5$ & $123.5 \pm 1.7$ & $133.0 \pm 4.8$ & $135.4 \pm 2.2$ & $135.8 \pm 4.7$ \\
\hline Condition factor ${ }^{b}$ & $1.47 \pm 0.03$ & $1.47 \pm 0.03$ & $1.52 \pm 0.04$ & $1.52 \pm 0.07$ & $1.35 \pm 0.05$ & $1.57 \pm 0.06$ \\
\hline \multicolumn{7}{|l|}{ Organ weight (mg) } \\
\hline Liver & $19.9 \pm 0.8$ & $20.6 \pm 1.5$ & $22.9 \pm 1.4$ & $18.1 \pm 2.2$ & $16.0 \pm 2.0$ & $21.0 \pm 3.3$ \\
\hline Kidney & $92.0 \pm 8.1$ & $107.9 \pm 10.6$ & $97.9 \pm 8.6$ & $134.3 \pm 33.0$ & $137.3 \pm 37.4$ & $193.5 \pm 40.4$ \\
\hline Intestine & $36.4 \pm 2.4$ & $37.3 \pm 2.0$ & $36.8 \pm 2.8$ & $86.5 \pm 8.4$ & $69.5 \pm 9.2$ & $89.6 \pm 9.7$ \\
\hline Muscle & $153.5 \pm 8.2$ & $175.0 \pm 9.3$ & $202.0 \pm 15.8$ & $168.7 \pm 23.3$ & $139.8 \pm 12.0$ & $135.3 \pm 8.9$ \\
\hline \multicolumn{7}{|c|}{$\begin{array}{l}\text { Organosomatic Index (g wet } \\
\text { organ/g body weight } \times 100 \% \text { ) }\end{array}$} \\
\hline $\mathrm{HSI}^{c}$ & $0.72 \pm 0.03$ & $0.71 \pm 0.05$ & $0.79 \pm 0.03$ & $0.50 \pm 0.02$ & $0.47 \pm 0.04$ & $0.52 \pm 0.03$ \\
\hline $\mathrm{KSI}^{c}$ & $3.42 \pm 0.31$ & $3.68 \pm 0.34$ & $3.38 \pm 0.23$ & $3.59 \pm 0.72$ & $3.98 \pm 0.97$ & $4.66 \pm 0.48$ \\
\hline $\mathrm{ISI}^{c}$ & $1.26 \pm 0.09$ & $1.30 \pm 0.08$ & $1.28 \pm 0.09$ & $2.41 \pm 0.13$ & $2.04 \pm 0.24$ & $2.27 \pm 0.14$ \\
\hline $\mathrm{MSI}^{c}$ & $5.56 \pm 0.43$ & $6.04 \pm 0.23$ & $7.07 \pm 0.52$ & $4.71 \pm 0.51$ & $4.23 \pm 0.51$ & $3.56 \pm 0.43$ \\
\hline
\end{tabular}

${ }^{a}$ Insulin or alloxan were injected into animals at a dose of $100 \mathrm{ng}$ and $0.2 \mathrm{mg}$ per gram of body weight, respectively; control animals received $0.6 \%$ saline. Data are presented as means \pm SEM.

${ }^{b}$ Calculated as [body weight $\left.(\mathrm{g}) /(\text { body length, } \mathrm{mm})^{3}\right] \times 10^{6}$.

${ }^{c}$ Abbreviations: HSI, hepatosomatic index; KSI, kidney-somatic index; ISI, intestinal-somatic index; MSI, muscular-somatic index.

compared to controls. The rate of lipolysis was, unexpectedly, significantly decreased in liver, muscle, and intestine after alloxan administration to larvae. In stage 6 metamorphosing lampreys, INS tended to decrease the rate of lipolysis; lipase activity was significantly reduced in liver. Alloxan treatment of stage 6 transformers generally did not affect lipase activity, except in liver which displayed significantly lower activity compared to that of control animals.

\section{The Rate of de Novo Fatty Acid Synthesis}

The rate of de novo FA synthesis was assessed by changes in ACC activity (Fig. 4). Treatment of larvae with INS tended to increase the rates of FA synthesis of kidney, muscle, and intestine. Treatment of larvae with alloxan generally tended to lower rates of FA synthesis in these tissues; alloxan treatment significantly reduced ACC activity in larval lamprey. In stage 6 metamorphosing animals, the rate of FA synthesis was increased significantly in the kidney and liver after INS administration. Treatment with alloxan resulted in no significant change in the rate of FA synthesis in the various tissues.

\section{The Rate of Triacylglycerol Synthesis}

The rate of TG synthesis was assessed by DGAT activity (Fig. 5). In larvae, the rate of TG synthesis tended to increase in kidney and muscle after INS administration. Renal DGAT activity was significantly lower in alloxan-treated animals compared to the INS-treated group, although enzyme activity was not different from that in controls. In stage 6 metamorphosing animals, INS injection increased rates of TG synthesis in liver and muscle; this response was significant in muscle. Following alloxan injection, a pronounced decrease in the rate of renal TG synthesis was observed. The rates of TG synthesis in the liver and muscle also tended to decrease with alloxan treatment.

\section{Immunocytochemistry}

In all animals, the anti-bINS immunostained all of the cells within the submucosal islets and specific clumps of cells within the mucusa of the alimentary canal at the junction of the anterior intestine and esophagus. The distribution of immunoreactivity for $\beta$ cells was similar to that previously described for this species (Elliot and Youson, 1986; Cheung et al., 1991). 


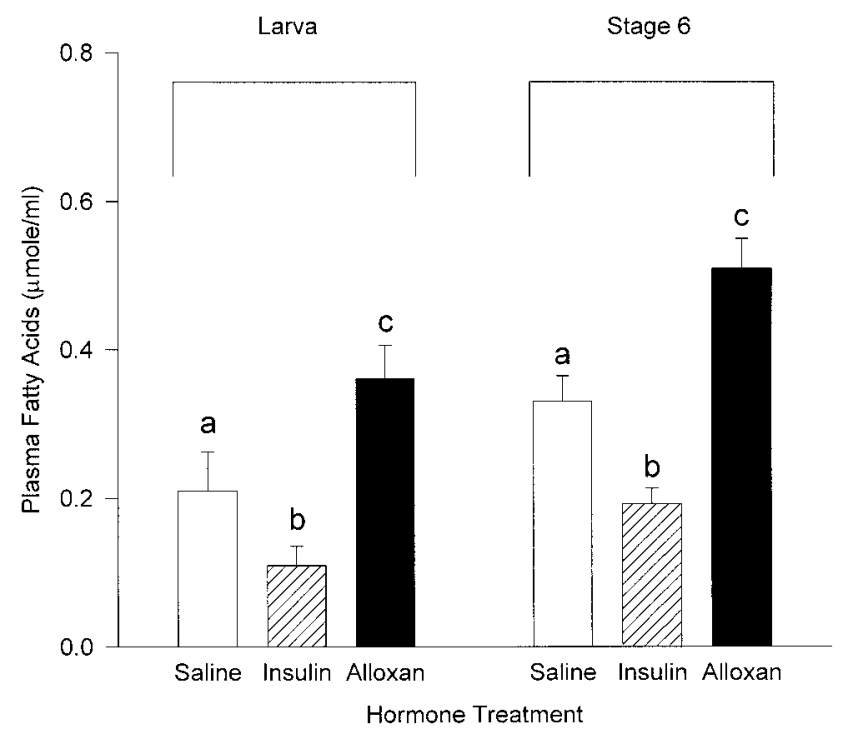

FIG. 1. Effects of insulin and alloxan on plasma fatty acid concentrations in the larvae $(n=10$ at each treatment) and stage $6(n=5$ at each treatment) metamorphosing lamprey, Petromyzon marinus. Data are expressed as means \pm SEM. For each developmental stage, groups with different letters are significantly different $(P<0.05)$ from each other.

There was no visible difference in immunoreactivity between $\beta$ cells of alloxan-treated larvae and those from the two controls (untreated and saline-injected controls, data not shown).

\section{DISCUSSION}

The results of this study indicate that INS modulates lipid metabolism in larval and metamorphosing lamprey. The specific effects of INS were tissue and development dependent. Insulin-stimulated lipid deposition proceeded from the synthesis of fatty acid and triacylglycerol in storage sites and resulted in depressed plasma FA levels. Insulin also inhibited lipid breakdown.

We report for the first time that INS lowers plasma FA in both larval and metamorphosing lampreys. The hypolipidemic action of INS observed in this study is consistent with that reported for adult lamprey (Plisetskaya and Mazina, 1969), salmonids (Harmon and Sheridan, 1992), amphibians (Penhos et al., 1967), and mammals (for review, see Epple and Brinn, 1987), but is different from the lack of a hypolipidemic effect reported in birds (Epple and Brinn, 1987). The levels of plasma FA in larvae and stage 6 metamorphosing $P$. marinus observed in this study were relatively lower than those reported in spawning adult $P$. marinus (John et al., 1977; Sower et al., 1985) and in adult L. fluviatilis (Plisetskaya and Mazina, 1969). The difference may be due to diet, because the larvae of both species are benthic filter-feeding detritovores while their adult counterparts are pelagic parasitic carnivores (Hardisty, 1979). It should be noted that changes in plasma FA levels of lamprey may also vary with other environmental and physiological cues (e.g., other hormones) as described by Plisetskaya (1980).

Insulin has a lipid anabolic effect on larval lampreys. This conclusion is supported by several observations. First, INS injection resulted in pronounced increases in the total lipid concentration of kidney and muscle, primary sites for lipid accumulation in $P$. marinus (Youson et al., 1979). The increased renal and muscular lipid concentration was supported by a lower rate of lipolysis as well as by a higher rate of lipogenesis, as indicated by FA synthesis and TG synthesis. The in vivo antilipolytic activity of INS in larval lamprey lipid depots, e.g., kidney, liver, and muscle, is consistent with that reported in rainbow trout liver (Harmon and Sheridan, 1992) and in mammal adipose tissue (Fain, 1980). Lower hepatic rates of FA synthesis after INS administration is unexpected, but possibly results from increased hepatic rate of cholesterol synthesis, a process which competes for acetyl-CoA substrate. This explanation is supported by previous studies that showed that larval lamprey liver contains considerable amounts of cholesterol (Kao et al., 1997a) when the bile duct system exists (Youson, 1980) and that INS stimulated cholesterol synthesis in mammal liver (Brindley and Lawson, 1983). The lack of an effect of INS on hepatic TG synthesis in larval lamprey was also unexpected. However, INS failed to stimulate TG synthesis in rat adipocytes as well (Sooranna and Saggerson, 1978), principally because substrates were used for phospholipid (PL) synthesis (Brindley and Lawson, 1983). Whether or not INS stimulates PL synthesis in larval lamprey remains to be determined. It also should be noted that the liver is not a primary lipid depot in P. marinus except under conditions of starvation (cf. Youson, 1983). 


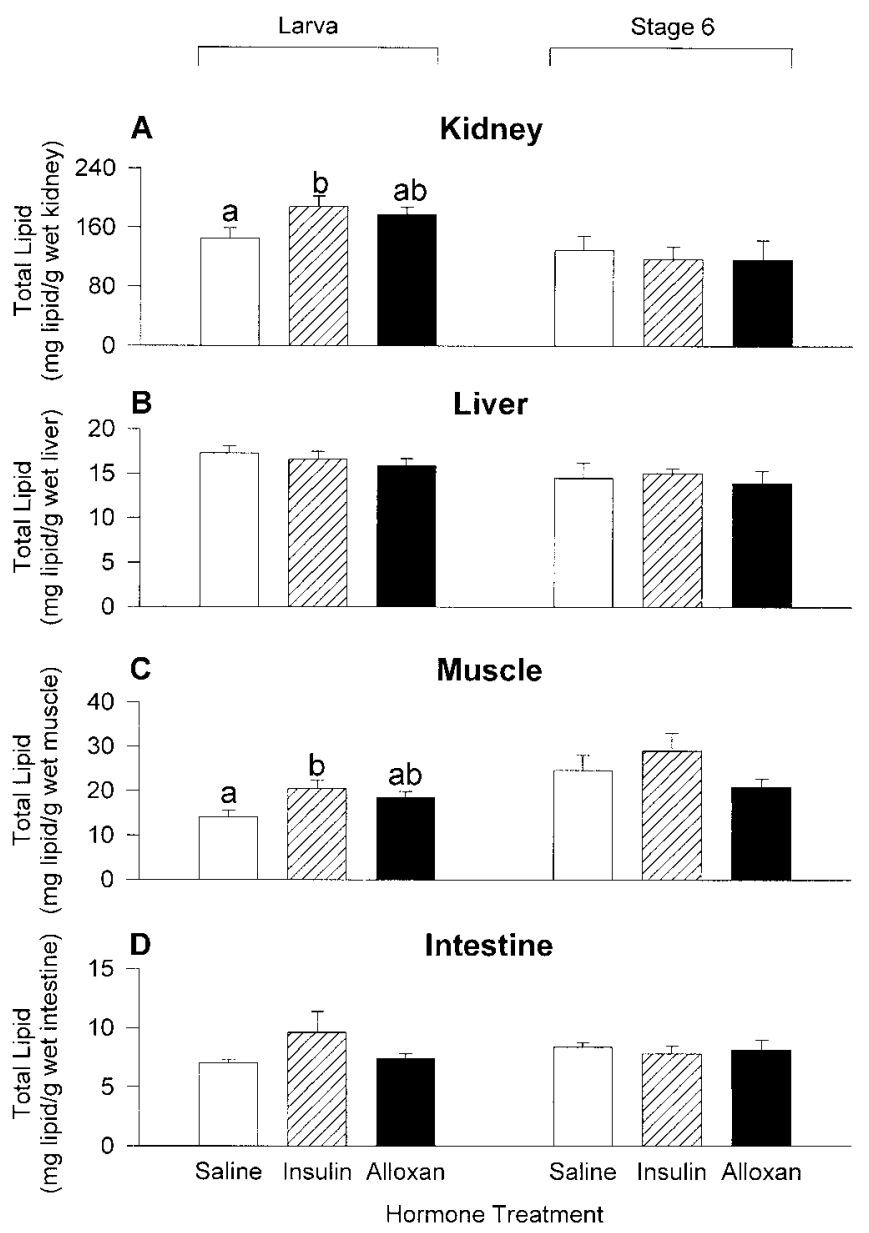

FIG. 2. Effects of insulin and alloxan on lipid concentration in the (A) kidney, (B) liver, (C) muscle, and (D) intestine of larvae $(n=10$ at each treatment) and stage $6(n=5$ at each treatment) metamorphosing lampreys, $P$. marinus. Data are expressed as means \pm SEM. For each developmental stage, groups with different letters are significantly different $(P<0.05)$ from each other.

Second, alloxan injection resulted in elevated plasma FA levels in larval lamprey, enhanced rates of lipolysis in kidney, and reduced rates of TG synthesis in kidney. Increased renal lipid mobilization accounts for decreased renal lipid deposition and elevated plasma FA levels. Because TG is the dominant lipid class in the kidney (Kao et al., 1997a), hydrolysis of stored renal TG into FA could supply FA for $\beta$ oxidation in liver and muscle and for reesterification of FA to TG and phospholipid in liver, intestine, and, possibly, muscle. This suggestion may explain why liver, muscle, and intestine displayed relatively lower rates of lipolysis and unchanged rates of TG synthesis in alloxan-treated larvae compared to control animals. Fatty liver in diabetic mammals has been reported (Brindley and
Lawson, 1983). Lower rates of FA synthesis in lipid depots and intestine are presumably due to decreased uptake of plasma glucose and FA (which are responsible for the supply of substrates for lipogenesis) and/or due to increased $\beta$ oxidation (which is the reverse pathway of FA synthesis). Another explanation is that increased plasma FA may allosterically inhibit ACC activity, as reported in mammals (Saggerson, 1980).

Third, immunoneutralization of lamprey INS by injection of anti-lamprey-INS resulted in elevated plasma FA in larvae accompanied by reduced renal total lipid concentration and by an enhanced rate of hepatic lipolysis, as indicated by increased TGL activity (Youson et al., 1992). Together, these findings sug- 
gest that the elevated levels of plasma INS observed in larval lampreys (Youson et al., 1994) play a role in directing the accumulation of lipid in premetamorphic larvae (Lowe et al., 1973; O'Boyle and Beamish, 1977; Youson et al., 1979) and in animals in the early stages of metamorphosis (Kao et al., 1997a,b) by enhancing lipogenesis and reducing lipolysis in depots sites (O'Boyle and Beamish, 1977; Kao et al., 1997b).

Insulin also seems to play a lipid anabolic role in metamorphic lampreys, although the nature of the responses were somewhat different from those displayed by larvae. These differences were not unex-
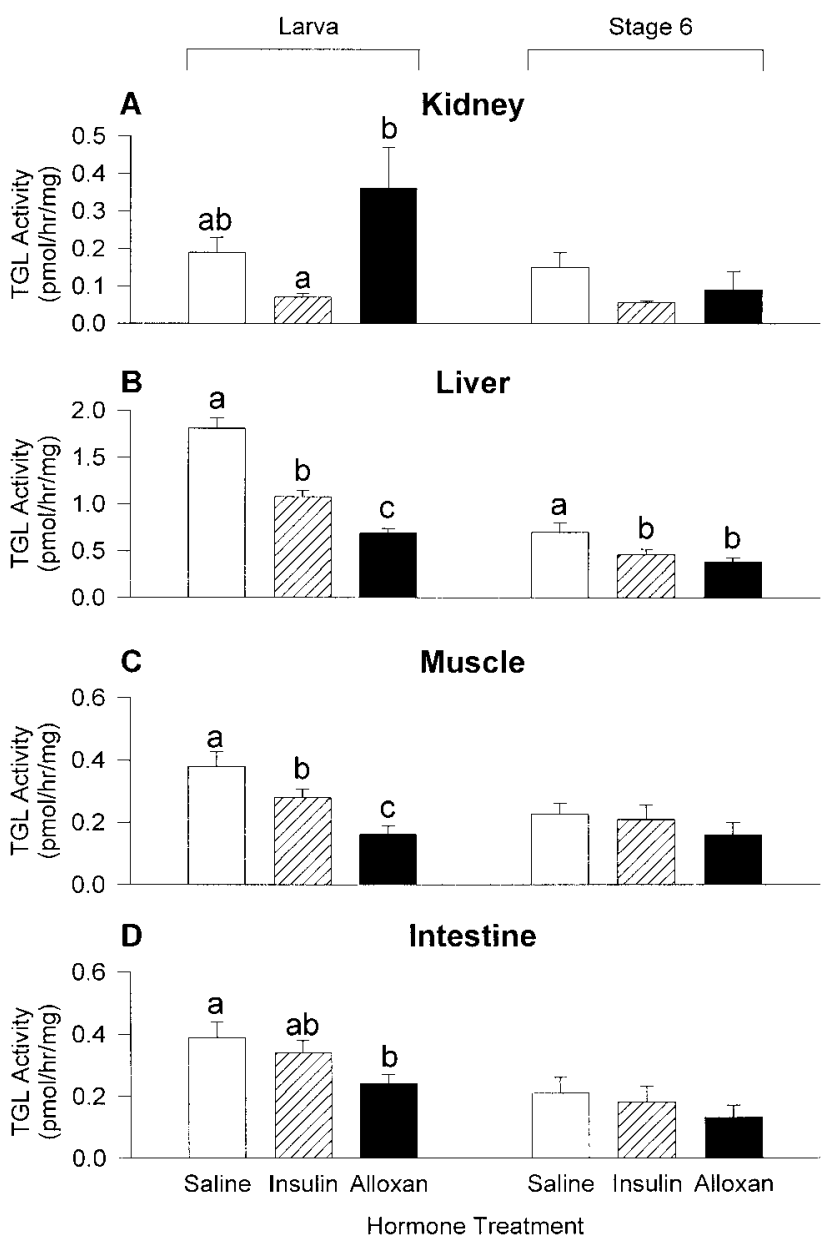

FIG. 3. Effects of insulin and alloxan on the rate of lipolysis, as indicated by triacylglycerol (TG) lipase activity, in the (A) kidney, (B) liver, (C) muscle, and (D) intestine of larvae $(n=10$ at each treatment) and stage $6(n=5$ at each treatment) metamorphosing lampreys, $P$. marinus. Data are expressed as means \pm SEM. For each developmental stage, groups with different letters are significantly different $(P<0.05)$ from each other.
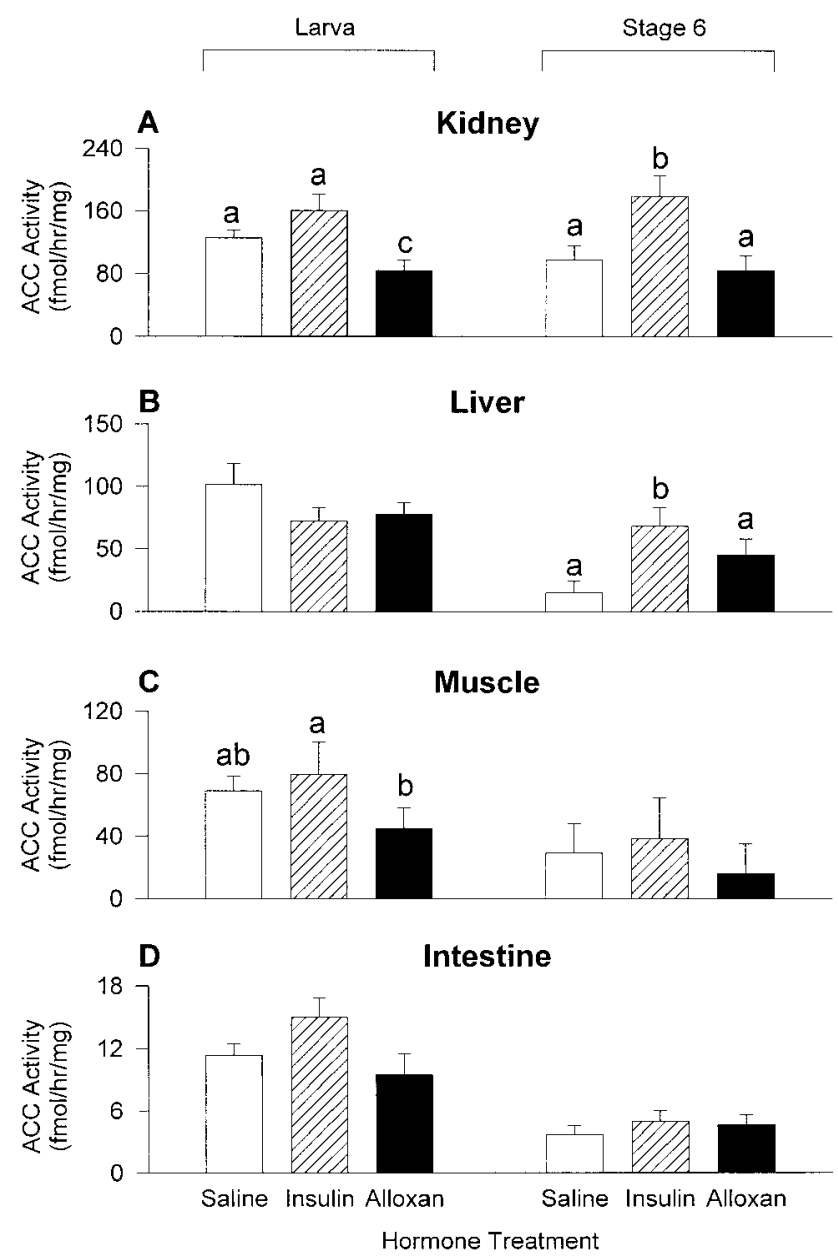

FIG . 4. Effects of insulin and alloxan on the rate of de novo fatty acid synthesis, as indicated by acetyl-CoA carboxylase (ACC) activity, in the (A) kidney, (B) liver, (C) muscle, and (D) intestine of larvae ( $n=10$ at each treatment) and stage $6(n=5$ at each treatment) metamorphosing lampreys, P. marinus. Data are expressed as means \pm SEM. For each developmental stage, groups with different letters are significantly different $(P<0.05)$ from each other.

pected because we have previously shown that larval and metamorphosing lamprey are in different phases of lipid metabolism (Kao et al., 1997a,b). Insulininduced reductions in plasma FA in metamorphosing animals was supported by decreased hepatic and renal lipolysis and by enhanced hepatic and renal FA synthesis. That alloxan treatment of stage 6 metamorphic lampreys resulted in hyperlipidemia accompanied by decreased renal TG synthesis and, to a lesser extent, decreased hepatic TG synthesis, is consistent with a lipid anabolic role of insulin. The basis for the difference in INS responsiveness between larval and stage 6 

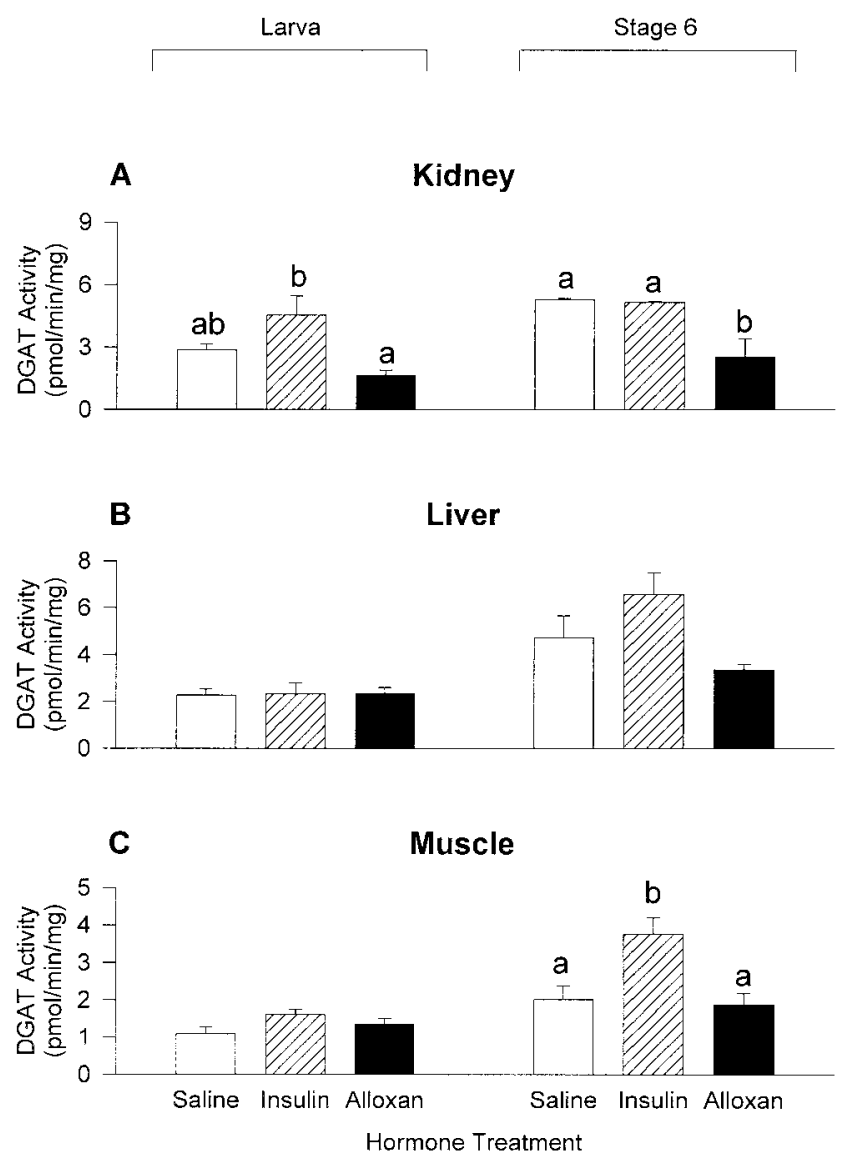

FIG. 5. Effects of insulin and alloxan on the rate of triacylglycerol synthesis, as indicated by diacylglycerol acyltransferase (DGAT) activity, in the (A) kidney, (B) liver, and (C) muscle of larvae $(n=10$ at each treatment) and stage $6(n=5$ at each treatment $)$ metamorphosing lampreys, P. marinus. Data are expressed as means \pm SEM. For each developmental stage, groups with different letters are significantly different $(P<0.05)$ from each other.

transformers (the later displaying less widespread antilipolytic effects and more widespread lipogenic effects than the former) is not known, but may be related to development-associated differences in INS receptor characteristics.

The observed alloxan-induced elevation of plasma FA is consistent with that reported in goldfish (Minick and Chavin, 1972) and in mammals (Dulin et al., 1983). We used alloxan in this study to provide additional insight into INS action and assumed that alloxan would alter intermediary metabolism by ablating INS. This assumption was supported by previous observations that alloxan damaged $\beta$ cells and increased plasma glucose in larval L. planeri (Morris and Islam,
1969) and adult L. fluviatilis (Bentley and Follett, 1965; Biuw, 1970). Many of our results, indeed, including those on plasma FA and plasma glucose (Kao et al., unpublished data), were consistent with INS disruption. However, alloxan injection in this study did not oppose all INS-induced alterations in lipid metabolism of larvae and stage 6 transformers. Moreover, there was no immediate cytotoxic effect of alloxan as assessed by the degree of INS immunostaining. In mammals, alloxan has been found to have a triphasic response on plasma glucose: (1) an initial hyperglycemia (1 to $4 \mathrm{~h}$ after injection), probably resulting from an initial inhibition of glucose-stimulated insulin release, followed by (2) a pronounced hypoglycemia (6 to $12 \mathrm{~h}$ after injection) stemming from insulin released from damaged $\beta$ cells, and finally, (3) a permanent hyperglycemia (evident 12-24 h after injection) (Cooperstein and Watkins, 1981). Marked species differences to alloxan treatment in terms of the development of hyperglycemia and $\beta$ cell toxicity have been observed. For example, islet lesions were difficult to define in sheep, pigeon, and frog (Lukens, 1948). Alloxan treatment has also been shown to have extra-islet modes of toxicity, including damage to kidney and liver tissue in lamprey (Morris and Islam, 1969; Biuw, 1970) and in mammals (Dulin et al., 1983); in mammals, alloxan was shown to alter the activities of a number of enzymes associated with hepatic lipid and protein metabolism as well as to contribute to hyperglycemia by promoting hepatic glycogenolysis and reducing renal clearance of glucose (Dulin et al., 1983). Our findings suggest that in the time frame of our experiment ( 2 days, $0.2 \mathrm{mg} / \mathrm{g}$ body weight), alloxan toxicity in larval and metamorphosing sea lampreys is primarily on extra-islet tissues and/or enzymes. It also is possible that the $\beta$ cells of sea lampreys were affected by alloxan, but the insulin in their secretory granules, or perhaps lysosomes, still retained immunoreactivity.

The mechanism by which INS modulates lipid deposition in lamprey is unknown, but it may be direct and/or indirect via interaction with other factors, such as thyroid hormones (THs) and somatostatins (SSs). The direct action of INS on lipogenesis is possibly through a dephosphorylation pathway, resulting in the activation of ACC and DGAT, as reported in mammals (Brindley and Lawson, 1983; Mabrouk et al., 1990). The 
direct action of INS on antilipolysis also is possibly through a dephosphorylation pathway that results in the inhibition of TGL, as reported in rainbow trout (Harmon et al., 1993) and mammals (Fain, 1980).

The interaction of INS with other hormones is supported by several lines of evidence. First, acute immunoneutralization of lamprey INS resulted in decreased concentrations of plasma thyroxine and triiodothyronine in larvae (Youson et al., 1992). Second, treatment with THs, which blocked potassium perchlorate $\left(\mathrm{KCIO}_{4}\right.$, a goitrogen)-induced lamprey metamorphosis, completely reversed the $\mathrm{KCIO}_{4}$-induced lipid depletion pattern (Kao et al., 1996). Third, higher levels of plasma INS in larvae are consistent with their higher levels of plasma THs (Youson et al., 1994) and with accumulated total lipid in the body (Lowe et al., 1973) and depot organs (O'Boyle and Beamish, 1977; Kao et al., 1997a). Fourth, acute immunoneutralization of lamprey SS-14 and lamprey SS-34 resulted in increased plasma INS levels (Youson et al., 1992; unpublished data) in larvae. Fifth, SS-14 injection resulted in a metamorphosis-like lipid catabolic pattern along with hyperlipidemia (Kao et al., 1998). Finally, lower levels of intestinal-pancreatic SS concentration (Elliott and Youson, 1991) from larvae to stage 4 transformers of sea lamprey, $P$. marinus, was observed to coincide with a higher level of plasma INS in larvae. We speculate that INS and THs have a positive synergistic effect on lipid accumulation in larval lamprey. Such a synergism between INS and THs would erode in metamorphic lampreys as plasma levels of TH decline (Youson et al., 1994) and as the concentration of SSs increase. Other hormones, such as catecholamines (Plisetskaya and Mazina, 1969) and arginine vasotocin (John et al., 1977), also could be involved in regulating lipid metabolism in larvae and metamorphic lampreys (for review see Plisetskaya, 1980).

In summary, we have shown that INS modulates lipid metabolism of larval and metamorphosing lamprey. Insulin-induced lipogenesis and antilipolysis is similar to the pattern of lipid metabolism (phase I) displayed by lamprey during their spontaneous metamorphosis (Kao et al., 1997a,b) and suggests that INS plays a role, possibly in concert with other factors, in coordinating metamorphosis-associated changes in lipid metabolism.

\section{ACKNOWLEDGMENTS}

We gratefully acknowledge R. G. Manzon and J. A. Heinig (University of Toronto) for their technical help. This work was supported by grants from the National Science Foundation, USA (OSR 9452892 and IBN 9723058) to M. A. S., from the Great Lakes Fishery Commission to J. H. Y. and J. A. H., and from the Natural Sciences and Engineering Research Council of Canada (A5945) to J. H. Y.

\section{REFERENCES}

Bentley, P. J., and Follett, B. K. (1965). The effects of hormones on the carbohydrate metabolism of the lamprey Lampetra fluviatilis. J. Endocrinol. 31, 127-137.

Biuw, L. W. (1970). Alloxan effects on blood glucose level and pancreatic islet tissue in Lampetra fluviatilis. Gen. Comp. Endocrinol. 15, 43-51.

Bradford, M. M. (1976). A rapid and sensitive method for the quantitation of microgram quantities of protein utilizing the principle of protein-dye binding. Anal. Biochem. 72, 248-254.

Brindley, D. N., and Lawson, N. (1983). Control of triglyceride synthesis. In "The Adipocyte and Obesity: Cellular and Molecular Mechanisms" (A. Angel, C. H. Hollenberg, and D. A. K. Roncari, Eds.), pp. 155-164. Raven Press, New York.

Cheung, R., Ferreira, L. C. G., and Youson, J. H. (1991). Distribution of two forms of somatostatin and peptides belonging to the pancreatic polypeptide family in tissues of larval lampreys, Petromyzon marinus L.: An immunohistochemical study. Gen. Comp. Endocrinol. 82, 93-102.

Cooperstein, S. J., and Watkins, D. (1981). Action of toxic drugs on islet cells. In "The Islets of Langerhans. Biochemistry, Physiology, and Pathology" (S. J. Cooperstein and D. Watkins, Eds), pp. 387-425. Academic Press, New York.

Dulin, W. E., Gerritsen, G. C., and Chang, A. Y. (1983). Experimental and spontaneous diabetes in animals. In "Diabetes Mellitus: Theory and Practice" (M. Ellenberg and H. Rifkin, Eds.), 3rd ed., pp. 361-407. Elsevier/North Holland, New York.

Elliott, W. M., and Youson, J. H. (1986). Immunocytochemical localization of insulin and somatostatin in the endocrine pancreas of the sea lamprey, Petromyzon marinus L. Cell Tissue Res. 243, 629-634.

Elliott, W. M., and Youson, J. H. (1991). Somatostatin concentrations in the pancreatic-intestinal tissues of the sea lamprey, Petromyzon marinus L., at various periods of its life cycle. Comp. Biochem. Physiol. 99A, 357-360.

Epple, A., and Brinn, J. E. (1987). “The Comparative Physiology of the Pancreatic Islets." Springer-Verlag, New York.

Fain, J. N. (1980). Hormonal regulation of lipid mobilization from adipose tissue. In "Biochemical Actions of Hormones" (G. Litwack, Ed.), Vol. VII, pp. 119-204. Academic Press, New York. 
Hardisty, M. W. (1979). “The Biology of the Cyclostomes." Chapman and Hall Ltd., London.

Harmon, J. S., and Sheridan, M. A. (1992). Effects of nutritional state, insulin, and glucagon on lipid mobilization in rainbow trout, Oncorhynchus mykiss. Gen. Comp. Endocrinol. 87, 214-221.

Harmon, J. S., Rieniet, L. M., and Sheridan, M. A. (1993). Glucagon and insulin regulate lipolysis in trout liver by altering phosphorylation of triacylglycerol lipase. Am. J. Physiol. 265, R255-R260.

John, T. M., Tomas, E., George, J. C., and Beamish, F. W. H. (1977). Effect of vasotocin on plasma free fatty acid level in the migrating anadromous sea lamprey. Archs. Int. Physiol. Biochim. 85, 865-870.

Kao, Y.-H., Youson, J. H., Manzon, R. G., and Sheridan, M. A. (1996). Role of thyroid hormones on lipid metabolism of lamprey (Petromyzon marinus) associated with metamorphosis. Am. Zool. 36, 97A.

Kao, Y.-H., Youson, J. H., and Sheridan, M. A. (1997a). Differences in the total lipid and lipid class composition of larvae and metamorphosing sea lamprey, Petromyzon marinus. Fish Physiol. Biochem. 16, 281-290

Kao, Y.-H., Youson, J. H., Holmes, J. A., and Sheridan, M. A. (1997b). Changes in lipolysis and lipogenesis in selected tissues of the landlocked lamprey, Petromyzon marinus, during metamorphosis. J. Exp. Zool. 277, 301-312.

Kao, Y.-H., Youson, J. H., Holmes, J. A., and Sheridan, M. A. (1998). Effects of somatostatin on lipid metabolism of larvae and metamorphosing landlocked sea lamprey, Petromyzon marinus. Gen. Comp. Endocrinol. 111, 177-185.

Lowe, D. R., Beamish, F. W. H., and Potter, I. C. (1973). Changes in the proximate body composition of the landlocked sea lamprey Petromyzon marinus (L.) during larval life and metamorphosis. J. Fish Biol. 5, 673-682.

Lukens, F. D. W. (1948). Alloxan diabetes. Physiol. Rev. 28, 304-330.

Mabrouk, G. M., Helmy, I. M., Thampy, K. G., and Wakil, S. J. (1990). Acute hormonal control of acetyl-CoA carboxylase: The roles of insulin, glucagon, and epinephrine. J. Biol. Chem. 265, 6330-6338.

Minick, M. C., and Chavin, W. (1972). Effects of alloxan, streptozotocin or D-mammoheptulose upon serum free fatty acid and serum glucose levels in goldfish, Carassius auratus L. Comp. Biochem. Physiol. 42B, 367-376.

Morris, R., and Islam, D. S. (1969). The effect of hormones and hormone inhibitors on blood sugar regulation and the follicles of Langerhans in ammocoete larvae. Gen. Comp. Endocrinol. 12, 81-90.

Noma, A., Okabe, H., and Kita, M. (1973). A new colorimetric micro-determination of free fatty acids in serum. Clin. Chim. Acta 43, 317-320.

O'Boyle, R. N., and Beamish, F. W. H. (1977). Growth and intermedi- ary metabolism of larval and metamorphosing stages of the landlocked sea lamprey, Petromyzon marinus L. Env. Biol. Fish. 2, 103-120.

Penhos, J. C., Uno, B., and Houssay, B. A. (1967). Glucose and lipid metabolism in the toad's perfused liver. Gen. Comp. Endocrinol. 8, 297-304.

Plisetskaya, E. M. (1980). Fatty acid levels in blood of cyclostomes and fish. Environ. Biol. Fish. 5, 273-290.

Plisetskaya, E. M., and Mazina, T. I. (1969). The influence of hormones on nonesterified fatty acids in the blood of the Baltic lamprey, Lampetra fluviatilis. J. Evol. Biochem. Physiol. 5, 374-378.

Saggerson, E. D. (1980). Regulation of lipid metabolism in adipose and liver cells. In "The Biochemistry of Cellular Regulation, Vol. II. Clinical and Scientific Aspects of the Regulation of Metabolism" (M. Ashwell, Ed.), pp. 207-256. CRC Press, Boca Raton, FL.

Sheridan, M. A., and Kao, Y.-H. (1998). Regulation of metamorphosisassociated changes in the lipid metabolism of selected vertebrates. Am. Zool. 38, 350-368.

Sooranna, S. R., and Saggerson, E. D. (1978). A decrease in diacylglycerol acyltransferase after treatment of rat adipocytes with adrenaline. FEBS Lett. 9, 85-87.

Sower, S. A., Plisetskaya, E. M., and Gorbman, A. (1985). Changes in plasma and thyroid hormones and insulin during final maturation and spawning of the sea lamprey, Petromyzon marinus. Gen. Comp. Endocrinol. 58, 259-269.

Youson, J. H. (1980). Morphology and physiology of lamprey metamorphosis. Can. J. Fish. Aquat. Sci. 37, 1687-1710.

Youson, J. H. (1983). Effects of starvation and iron concentration and deposition in tissues of larval lampreys, Petromyzon marinus L.: Morphological and quantitative analyses. Can. J. Zool. 71, 20182032.

Youson, J. H., Lee, J., and Potter, I. C. (1979). The distribution of fat in larval, metamorphosing and young adult anadromous sea lamprey, Petromyzon marinus L. Can. J. Zool. 57, 237-246.

Youson, J. H., Leatherland, J. F., Plisetskaya, E. M., and Sheridan, M. A. (1992). "Metabolic and Hormonal Changes in Larval Lampreys (Petromyzon marinus) Following Acute Neutralization with Anti-insulin and Anti-somatostatin," 2nd International Symposium on Fish Endocrinology. Saint-Malo, France.

Youson, J. H., Plisetskaya, E. M., and Leatherland, J. F. (1994). Concentrations of insulin and thyroid hormones in the serum of landlocked sea lampreys (Petromyzon marinus) of three larval year classes, in larva exposed to two temperature regimes, and in individuals during and after metamorphosis. Gen. Comp. Endocrinol. 94, 294-304. 